\title{
Magnetic-Field Induced Isotropic-Nematic Phase Transition in PDLC Doped with Magnetic Nanoparticles
}

\author{
Z. Mitróová ${ }^{a, *}$, N. Tomašovičová ${ }^{a}$, M. Timko $^{a}$, M. KonerackÁ $^{a}$, L. TomČO $^{b}$, \\ J. JADŻYN ${ }^{c}$, T. PALEWSKI ${ }^{d}$, AND P. KOPČANSKY $\dot{Y}^{a}$ AND O.V. KOVALChUK ${ }^{e}$ \\ ${ }^{a}$ Institute of Experimental Physics, SAS, Watsonova 47, 04001 Košice, Slovakia \\ ${ }^{b}$ Faculty of Aeronautics, Technical University, Rampová 7, 041 21, Košice, Slovakia \\ ${ }^{c}$ Institute of Molecular Physics, Polish Academy of Sciences, M. Smoluchowskiego 17, 60-179 Poznań, Poland \\ ${ }^{d}$ International Laboratory of High Magnetic Fields and Low Temperatures, Polish Academy of Sciences \\ Gajowicka 95, 53-421 Wrocław, Poland \\ ${ }^{e}$ Institut of Physics, NASU, Kyiv, Ukraine
}

\begin{abstract}
The paper presents a study of the polymer dispersed liquid crystals that consist of liquid crystal 4 - trans- $^{\prime}{ }^{\prime}-n$ -hexyl-cyclohexyl-isothiocyanatobenzene (6CHBT) microdroplets dispersed in polyvinyl alcohol and doped with various kinds of magnetic particles. As magnetic nanoparticles there were used single walled carbon nanotubes and magnetite labeled single walled carbon nanotubes. The volume concentration of the particles was $2 \times 10^{-3}$. Magnetic properties were investigated by a SQUID magnetometer. The higher saturation magnetization has been achieved in sample polymer dispersed liquid crystal doped with magnetite labeled single walled carbon nanotubes. The phase transition temperature from isotropic to nematic phase at the external magnetic field $0 \mathrm{~T}$ and $12 \mathrm{~T}$ was monitored by precise capacitance measurements in the capacitance cell filled with prepared sample. The significant shift of the phase transition temperature $\left(0.2^{\circ} \mathrm{C}\right)$ at the external magnetic field $12 \mathrm{~T}$ has been observed in sample polymer dispersed liquid crystal doped with magnetite labeled single walled carbon nanotubes.
\end{abstract}

PACS: 61.30.Gd, 75.30.Hx, 75.75.Fk, 77.84.Nh

\section{Introduction}

Polymer dispersed liquid crystals (PDLCs) are a novel class of optical composites [1-5], which consist of polymer and liquid crystal (LC) in an appropriate ratio. Micron sized nematic liquid crystal (NLC) droplets are uniformly dispersed in a transparent polymer matrix. The nematic texture of these droplets is randomly oriented with respect to the neighbouring droplets such that incoming light into the cell is scattered, and the PDLC appears opaque. When an electric field is applied across the cell, the liquid crystal droplets align and the PDLC is transparent if the ordinary index of refraction is matched to the index of the polymer. The voltage and speed at which the PDLC switches from opaque to transparency and the optical contrast between the opaque and transparent state is controlled by the size, shape, and anchoring energy of the liquid crystal droplets, as well as the dielectric anisotropy and viscosity of the liquid crystal.

In the past decade, PDLCs have found great interest because of their promising use in advanced optical device applications, such as large flexible displays, switchable windows or paper-like displays for electronic books.

* corresponding author; e-mail: mitro@saske.sk
In our previous work [6] we studied dielectric properties of PDLCs doped with spherical and rod like nanoparticles. The goal of this work was to study the magnetic field induced isotropic- nematic phase transition in the PDLCs doped with single wall carbon nanotubes (SWCNT) and SWCNT functionalized with magnetite nanoparticles (so-called polymer dispersed ferronematics).

\section{Experimental}

The samples of PDLCs have been prepared by the following method. Liquid crystal (6CHBT) amount of $0.05 \mathrm{ml}$ was added to $5 \mathrm{ml}$ of $10 \%$ polyvinyl alcohol (PVA). This mixture was stirred at $10000 \mathrm{rpm}$ for $1 \mathrm{~min}$. A creamy white emulsion was obtained. It was let to degas and a thin bead was placed on a slide. After the water evaporation we got a thin film.

This technique was used also for preparing the PDLC films doped with various kinds of magnetic particles. SWCNT and magnetite labeled SWCNT (SWCNT/ $\left.\mathrm{Fe}_{3} \mathrm{O}_{4}\right)$ have been used as magnetic nanoparticles. The prepared samples are illustrated in Fig. 1.

The samples thickness was $50 \mu \mathrm{m}$. The structure of the films was investigated using the scanning electron microscope JSM-35 with an accelerating voltage of $35 \mathrm{keV}$. 


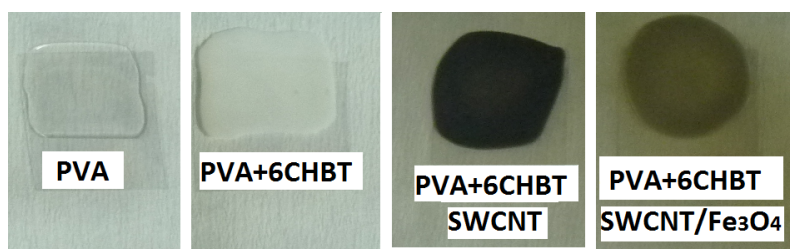

Fig. 1. The photo of the prepared samples.

To eliminate the effect of charging the surface, before the measurements a graphite film was deposited onto the surface of the PDLC film.

The magnetic properties of the samples were performed with a SQUID magnetometer (Quantum Design MPMS 5XL) up to $5 \mathrm{~T}$ at room temperature. The contribution of the 6CHBT in PVA was subtracted.

The phase diagram of the samples was obtained using the polarizing microscopy. Structural transitions in the samples were monitored by capacitance measurements. The capacitor was placed into a thermostated system. The distance between the electrodes (sample thickness) was $D=50 \mu \mathrm{m}$. The capacitance was measured at the frequency of $1 \mathrm{kHz}$ by the high precision capacitance bridge Andeen Hagerling.

\section{Results and discussion}

Polarizing microscopy analysis demonstrated that the size of 6CHBT microdroplets in the polymer matrix was from 5 to $8 \mu \mathrm{m}$. The magnetization properties of PDLCs doped with different nanoparticles are illustrated in Fig. 2. The saturation magnetization, $M_{\mathrm{S}}=$ $3.9 \times 10^{-3} \mathrm{~A} \mathrm{~m}^{2} \mathrm{~kg}^{-1}$, has been achieved in PDLC doped with $\mathrm{SWCNT} / \mathrm{Fe}_{3} \mathrm{O}_{4}$. The sample doped with SWCNT has lower value of saturation magnetization, $M_{\mathrm{s}}=7.4 \times 10^{-5} \mathrm{~A} \mathrm{~m}^{2} \mathrm{~kg}^{-1}$.

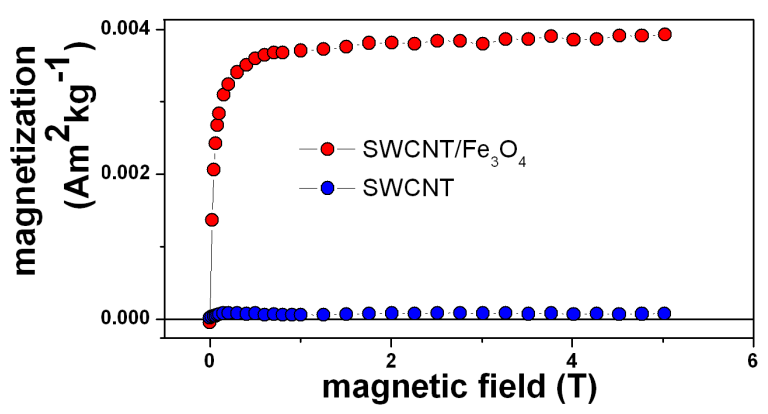

Fig. 2. Magnetic properties of the samples with volume concentration of magnetic nanoparticles $\Phi=2 \times$ $10^{-3}$ measured at room temperature.

During measurements of the temperature dependences of the capacitance, the magnetic field was applied parallel to the capacitor electrodes. The constant magnetic field was held, while the temperature was increased above the temperature of the transition from nematic to isotropic phase $T_{\mathrm{N}-\mathrm{I}}$ and then slowly decreased. The dependence of the measured capacitance reflects the re-orientation of the molecules of the liquid crystal. Figure 3 shows the temperature dependence of the capacitance of PDLC doped with SWCNT $/ \mathrm{Fe}_{3} \mathrm{O}_{4}$ measured without magnetic field and in magnetic field $12 \mathrm{~T}$. There is a significant shift in the temperature of isotropic to nematic phase transition due to applying the external magnetic field. In the case of doping liquid crystals with SWNCTs no measurable shift of the transition from isotropic to nematic phase after application of the external magnetic field $12 \mathrm{~T}$ was observed.

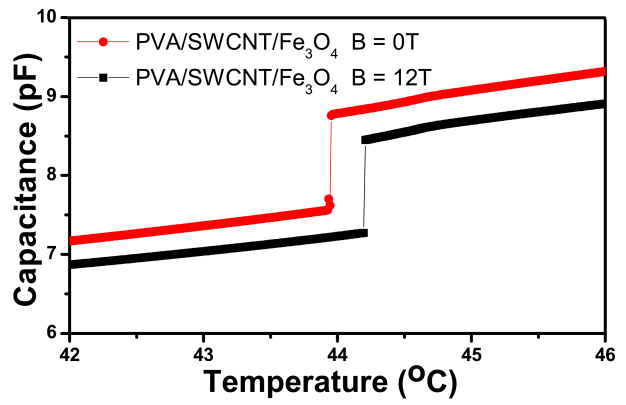

Fig. 3. The temperature dependence of capacitance of the samples with volume concentration of magnetic nanoparticles $\Phi=2 \times 10^{-3}$ measured without magnetic field and in magnetic field $12 \mathrm{~T}$.

It has long been known the possibility to substantially alter the nematic-isotropic transition temperature in liquid crystals with the external fields. However, the effect could not been induced by magnetic field $H$ until recently [7]. The principal reason is that the estimated critical fields are well over $100 \mathrm{~T}$ for traditional liquid crystal materials. The first experimental observation of the predicted magnetic-field dependence of the nematicisotropic phase transition temperature has been recently carried out [7] on a powerful electromagnet ( $H$ up to $30 \mathrm{~T}$ ). To demonstrate the effect, besides the powerful electromagnet, the proper choice of a "non-traditional" (bent-core) nematic liquid crystal material was also necessary. The "non-traditional" nematic material chosen in Ref. [7], has considerably different physical properties from "traditional" calamitic nematics. These properties, combined with the high magnetic field, have contributed to the observation of the phase transition temperature shift that was $\approx 0.8^{\circ} \mathrm{C}$ at the magnetic field of $30 \mathrm{~T}$, but at the magnetic field of $12 \mathrm{~T}$ the shift was about $0.25{ }^{\circ} \mathrm{C}[7]$.

In our sample doped with $\mathrm{SWCNT} / \mathrm{Fe}_{3} \mathrm{O}_{4}$, similar shift, about $0.2^{\circ} \mathrm{C}$, in the temperature of the phase transition from isotropic to nematic phase at the external magnetic field of $12 \mathrm{~T}$ was observed.

\section{Conclusions}

In this work we have studied PDLCs which have consisted of liquid crystal 6CHBT microdroplets dispersed 
in a PVA and doped with various kinds of magnetic nanoparticles. Magnetic measurements show ferromagnetic properties of the samples. The shift about $0.2^{\circ} \mathrm{C}$ in the temperature of the phase transition from isotropic to nematic phase at the external magnetic field of $12 \mathrm{~T}$ was observed in the PDLC doped with $\mathrm{SWCNT} / \mathrm{Fe}_{3} \mathrm{O}_{4}$.

\section{Acknowledgments}

This work was supported by the Slovak Research and Development Agency under the contract No. APVV-0171-10, by the projects Nos. 26220120021, 26220120033 in the frame of Structural Funds of European Union, Centre of Excellence of SAS Nanofluid and VEGA 0077.

\section{References}

[1] J.L. Fergason, SID Int. Symp. Dig. Tech. Pap. 16, 68 (1985).

[2] P.S. Drzaic, J. Appl. Phys. 60, 2142 (1986).

[3] J.W. Doane, N.A. Vaz, B.G. Wu, S. Zumer, Appl. Phys. Lett. 48, 269 (1986).

[4] N.A. Vaz, G.W. Smith, G.P. Montgomery, Mol. Cryst. Liq. Cryst. 146, 1 (1987).

[5] Pankaj Kumar, K.K. Raina, Curr. Appl. Phys. 7, 636 (2007).

[6] P. Kopčanský, M. Timko, Z. Mitróová, V. Závišová, M. Koneracká, N. Tomašovičová, L. Tomčo, O.P. Gornitska, O.V. Kovalchuk, V.M. Bykov, T.M. Kovalchuk, I.P. Studenyak, Semiconductor Physics, Quantum Electron. Optoelectron. 13, 343 (2010).

[7] T. Ostapenko, D.B. Wiant, S.N. Sprunt, A. Jakli, J.T. Gleeson, Phys. Rev. Lett. 101, 247801 (2008). 